Archives de sciences sociales des religions

172 | octobre-décembre

Bulletin Bibliographique

\title{
Yann Raison du Cleuziou, Qui sont les cathos aujourd'hui?
}

Paris, Desclée de Brouwer, 2014, 332 p.

Marion Maudet

\section{OpenEdition}

\section{Journals}

Édition électronique

URL : http://journals.openedition.org/assr/27440

DOI : $10.4000 /$ assr. 27440

ISSN : $1777-5825$

Éditeur

Éditions de l'EHESS

Édition imprimée

Date de publication : 1 octobre 2015

Pagination : 353

ISBN : 978-2-7132-2515-4

ISSN : 0335-5985

\section{Référence électronique}

Marion Maudet, «Yann Raison du Cleuziou, Qui sont les cathos aujourd'hui ? », Archives de sciences sociales des religions [En ligne], 172 | octobre-décembre, mis en ligne le 08 juin 2016, consulté le 23 septembre 2020. URL : http://journals.openedition.org/assr/27440 ; DOI : https://doi.org/10.4000/ assr. 27440

Ce document a été généré automatiquement le 23 septembre 2020

(C) Archives de sciences sociales des religions 


\section{Yann Raison du Cleuziou, Qui sont les cathos aujourd'hui?}

Paris, Desclée de Brouwer, 2014, 332 p.

Marion Maudet

\section{RÉFÉRENCE}

Yann Raison du Cleuziou, Qui sont les cathos aujourd'hui ?, Paris, Desclée de Brouwer, 2014, $332 \mathrm{p}$. 
1 Alors qu'on publie fréquemment des chiffres faisant état du déclin de l'appartenance à la religion catholique et de la pratique religieuse et que les discours autour de la sécularisation de la société française abondent, l'ouvrage Qui sont les cathos aujourd'hui? propose une enquête sociologique approfondie sur les " catholiques engagés » à l'heure actuelle. L'auteur s'intéresse aux catholiques dont l'engagement religieux est visible, à celles et ceux qui s'investissent dans une paroisse ou une association religieuse. Remettant en question la thèse de la "modernité ", qui serait à son sens surtout un "récit auto-entretenu», il souhaite partir de la «logique des acteurs» eux-mêmes (p. 8) pour mieux appréhender et comprendre la manière dont les catholiques vivent, pratiquent

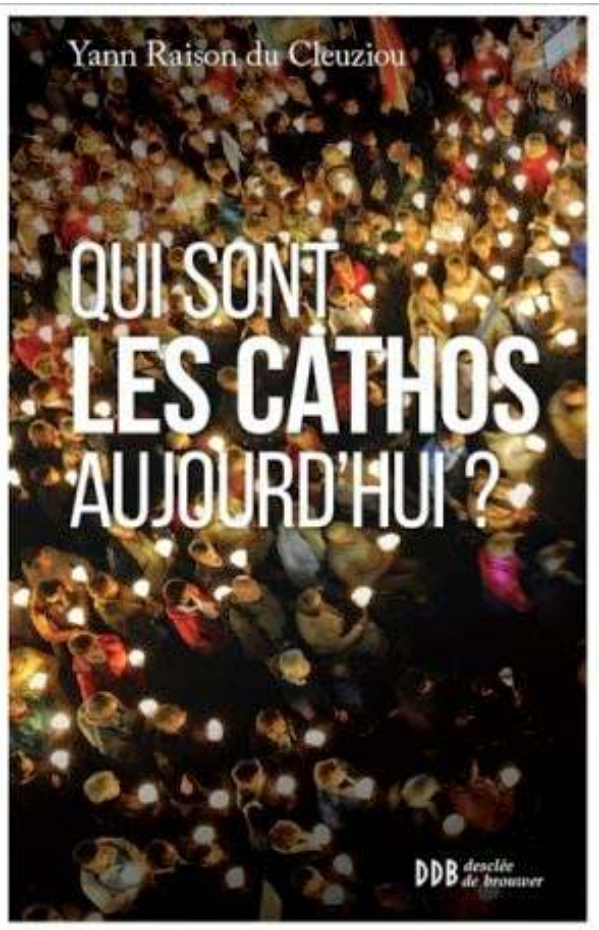
leur religion, perçoivent le monde qui les entourent tout autant que les évolutions de l'Église elle-même. L'ouvrage est le résultat d'une recherche collective initiée par l'association chrétienne «Confrontations ». Au total, 177 entretiens (collectifs et individuels) ont été menés, portant sur la période du pontificat de Benoît XVI; ils ont pour objet de donner une représentation de la diversité du catholicisme actuel. Les résultats sont présentés en deux parties: la première partie propose une description détaillée de douze portraits de catholiques tandis que, dans la seconde, l'auteur revient plus précisément sur les relations variées qu'entretiennent les catholiques avec leur Église. Comme le souligne l'auteur, la recherche ayant été menée avant l'élection du pape François, il est possible que des positions présentées aient pu évoluer, l'approche et le «style » du nouveau souverain pontife ayant «renversé bien des préventions à l'égard de la papauté, voire du catholicisme » (p. 295).

2 La première partie, écrite avec le concours de Geneviève Dahan-Seltzer et Françoise Parmentier, revient ainsi sur différents visages du catholicisme. Il s'agit de la présentation de "profils-types» de catholiques, construits à partir des données recueillies selon un certain nombre de "positions structurales » (p. 18). L'originalité de la démarche est ici double: tout d'abord, ce ne sont pas des profils réels qui sont présentés mais des profils reconstitués à partir des différents récits d'entretiens recueillis. Par ailleurs, à la fin de chaque description, est proposé un tableau récapitulatif des informations les plus importantes relatives à la socialisation de l'individu-type (principalement familiale), de son ancrage dans la foi (par le militantisme, les groupes de prières, les louanges, la communauté paroissiale, etc.), de sa posture vis-à-vis du monde, de sa perception de l'Église, de sa perception des autorités ecclésiales et de sa vision de l'avenir. Quatre grands groupes sont ainsi mis en évidence, qui se divisent ensuite en sous-groupes parfois en fort conflit sur certaines des positions évoquées plus haut. Les « inconciliables» (chapitre 1) décrivent leur foi 
en rapport avec le concile Vatican II, que ce soit pour valoriser certains renouvellements qu'il a engendrés (comme les "charismatiques ») ou à l'inverse pour critiquer le désordre qu'il a créé (position adoptée par les «traditionalistes»). Le deuxième groupe rassemble les «blessés de l'institution» (chapitre 2), qui opposent rapidement l'Église en tant qu'institution et l'Évangile. Ces catholiques regrettent les positions de l'Église sur un certain nombre de sujets qui excluent de nombreux fidèles (homosexuels, divorcés-remariés, femmes en responsabilité ecclésiales). Sont ensuite décrits les « artisans de la reconstruction » (chapitre 3). Ils interprètent la crise actuelle et le déclin du sentiment d'appartenance comme une période de transition, qui va permettre un renouvellement catholique salutaire. Cependant, si les «passeurs de frontières » valorisent une foi humble et la pluralité des formes du catholicisme, les «catholiques néoclassiques" empruntent à des formes appréciées comme «classiques » du catholicisme comme la soutane ou le col romain et souhaitent moins le dialogue avec le monde que l'affichage de la différence de leur identité catholique. Enfin, le dernier groupe, la «jeunesse sans complexe » (chapitre 4) se caractérise par une posture d'attente envers l'institution et une connaissance souvent approximative des textes et de l'histoire du catholicisme, sans que ces «jeunes » ne soient en conflit ouvert avec l'Église. Ici encore, ce groupe est traversé par de fortes différences, constitutives pour certaines d'entre elles de dissonances, notamment entre les «trentenaires libérés » qui adaptent les normes religieuses à leur situation personnelle et s'accommodent de certains dogmes (principalement sexuels), et les "jeunes sûrs d'eux-mêmes » qui valorisent le port d'attributs religieux visibles comme marqueurs identitaires et adoptent une attitude d'intransigeance vis-à-vis du respect des normes religieuses.

3 La deuxième partie de l'ouvrage s'intéresse aux relations qu'entretiennent les catholiques avec leur Église. L'auteur propose de nouvelles clés de lecture des douze portraits présentés en première partie afin de mieux comprendre ce que représente aujourd'hui "l'archipel catholique». Yann Raison du Cleuziou dégage quatre « nébuleuses catholiques » qui se répartissent autour de deux axes : un axe de modalité de la rencontre avec Dieu et un axe de modalité de mise en œuvre de la foi. Le premier axe oppose l'inspiration (rencontre non codifiée avec Dieu) et le culte (rencontre très codifiée), tandis que le second oppose la dévotion (engagement fort, pèlerinage, chants, louanges) et l'altruisme (œuvres de solidarité). Les nébuleuses ainsi mises en évidence (les inspirés - inspiration et dévotion, les observants - dévotion et culte, les concilaires revendiqués - culte et altruisme, et les émancipés - altruisme et inspiration) sont ensuite étudiées à l'aune de différentes questions structurantes: la messe et les attentes liturgiques (chapitre 5), le statut des prêtres et des laïcs (chapitre 6), la vision du sommet de l'institution (chapitre 7), l'identité médiatique de l'Église catholique (chapitre 8). Sur tous ces sujets, les différentes nébuleuses ont souvent des avis divergents, portant parfois sur la signification même de certains rites. Ainsi, si la messe reste une référence centrale pour les catholiques, leurs attentes à son sujet varient fortement et «ce déchirement [autour de la définition des modalités liturgiques des messes et des activités paroissiales] est d'autant plus violent que ce sont des conceptions de la vérité religieuse qui opposent les catholiques» (p.189). Les concilaires revendiqués souhaitent que la messe soit adaptée aux situations locales, qu'elle soit souple, centrée sur les moments de partage et l'accueil de tous (des petites filles au service d'autel à l'intégration des divorcés-remariés). Pour d'autres en revanche, comme les observants et une partie des inspirés, la messe doit être le lieu du 
respect de formes liturgiques d'inspiration monastique et ils regrettent leur superficialité.

4 De tous les clivages évoqués par l'auteur, le plus important semble être un conflit de générations entre l'avant et l'après Vatican II, qu'il faut selon lui inscrire dans les évolutions sociales fortes qui ont traversé la France du $\mathrm{xx}^{\mathrm{e}}$ siècle. En effet, "les aînés ont tout fait pour sortir du ghetto d'une religion trop sûre d'elle même et peu prompte à se remettre en question, quand les cadets cherchent les moyens de resserrer les rangs pour pallier la précarisation de leur groupe, retrouver une visibilité et s'affirmer en particulier face aux musulmans ou aux évangéliques » (p. 300).

5 Les thématiques évoquées par l'auteur sont multiples et proposent une vision claire et détaillée des avis et des représentations des catholiques, des liens qu'ils entretiennent (ou plus souvent, n'entretiennent pas) entre eux et de leurs espoirs, attentes et parfois frustrations vis-à-vis de l'institution. Cette recherche, appuyée sur de nombreuses références sociologiques précises, montre de manière éclairante à quel point le catholicisme (d') aujourd'hui est pluriel, voire divisé en "sous-cultures". Si cette situation n'est pas récente, ce qui l'est peut-être davantage est «la dérégulation qui affecte l'ensemble du catholicisme. Ces sous-cultures semblent s'autonomiser les unes par rapport aux autres, faute de régulation institutionnelle pour les faire converger ou pour les articuler » (p. 202). De nombreuses questions épineuses sont abordées, comme la place des femmes dans l'institution ecclésiale ou celle des divorcés-remariés. En revanche, si la place des homosexuels dans l'Église est plusieurs fois évoquée au cours de l'enquête, aucune personne homosexuelle n'a été interrogée, ce qui aurait pu ajouter à la précision d'un corpus d'entretiens déjà très complet.

6 Plusieurs typologies sont proposées par l'auteur (selon le rapport que les catholiques entretiennent à l'institution, à l'image médiatique de l'Église, à la vision de la société, etc.), mais si celles-ci témoignent d'un véritable souci d'exhaustivité dans les «logiques» actuelles du catholicisme, la multiplication des clés d'entrée en rend parfois la lecture assez délicate. On peut, par ailleurs, regretter que les groupes ainsi dégagés n'aient pas été décrits de manière encore plus précise tout particulièrement selon leurs caractéristiques sociodémographiques. L'âge est en effet un élément saillant pour l'interprétation, comme l'auteur le montre bien lui-même (les conciliaires revendiqués étant souvent plus âgés que la moyenne), mais la recherche aurait sans doute gagné à intégrer, notamment, une perspective de genre. Pour ne prendre qu'un exemple, si les "femmes en responsabilité ecclésiale" critiquent leur subordination dans l'institution, les arguments avancés (et présentés dans les extraits d'entretien) intègrent souvent une perspective essentialiste des rôles féminins/masculins (p. 77).

7 Enfin, la problématique de la recherche doit être rappelée : il s'agit de s'interroger sur la place des catholiques «engagés » en ne traitant volontairement pas de la situation des « consommateurs intermittents de la ritualité catholique» (p. 19). Autrement dit, alors que l'engagement religieux diminue et que seuls $4,5 \%$ assistent encore à la messe chaque dimanche, il est opportun de s'interroger sur la part des catholiques représentés dans l'ouvrage, qui traite moins des «catholiques aujourd'hui » que d'une frange minoritaire de ces derniers. L'orientation de la recherche est, de plus, fortement centrée sur le catholicisme en tant qu'institution. Assez peu d'informations sont données sur la manière dont les catholiques agissent dans le monde ou se positionnent sur des sujets de société. Quand ceux-ci sont abordés (comme la place des femmes ou 
celle des homosexuels), c'est toujours en lien avec l'Église et ces positions ne sont pas (suffisamment) structurantes en tant que telles.

8 Pour conclure, au-delà de ces limites, nous recommandons la lecture de cet ouvrage, éclairant tant sur la méthode des portraits fictifs que sur le travail de synthèse fourni, précis et détaillé. 